Check for updates

Cite this: Chem. Commun., 2020 56,4886

Received 15th January 2020,

Accepted 23rd March 2020

DOI: $10.1039 / \mathrm{d} 0 \mathrm{cc} 00327 \mathrm{a}$

rsc.li/chemcomm

\section{Structural elucidation of the degradation mechanism of nickel-rich layered cathodes during high-voltage cycling $\dagger$}

\author{
Jing Lai, ${ }^{a}$ Jun Zhang, ${ }^{b}$ Zuowei Li, ${ }^{a}$ Yao Xiao, ${ }^{a}$ Weibo Hua, (D)*c Zhenguo Wu, (D)*a \\ Yanxiao Chen, (D) ${ }^{a}$ Yanjun Zhong, (D) ${ }^{a}$ Wei Xiang ${ }^{d}$ and Xiaodong Guo ${ }^{a}$
}

\begin{abstract}
Phase transition occurring during cycling plays a fundamentally important role in the cycling performance of nickel-rich cathodes. Here, splitting of two $\mathrm{O} 3$ phases, rather than the often observed 01 phases in the conventional $\mathrm{LiCoO}_{2}$ electrode, was discovered in $\mathrm{LiNi}_{0.85} \mathrm{Co}_{0.10} \mathrm{Mn}_{0.05} \mathrm{O}_{2}$ at a high-voltage region (>4.6 V). Such degradation could be mitigated via Al doping.
\end{abstract}

Lithium ion batteries (LIBs) are considered one of the most promising energy storage devices for battery electric vehicles (BEVs). Among the available cathode materials for LIBs, nickelrich layered $\mathrm{LiNi}_{x} \mathrm{Co}_{y} \mathrm{M}_{1-x-y} \mathrm{O}_{2}(x \geq 0.8, \mathrm{M}=\mathrm{Mn}, \mathrm{Al}, \mathrm{Fe}, \mathrm{Mg}$, etc.) oxides are the ideal choice due to their higher capacity, lower cost and less environmental impact. ${ }^{1-4}$ However, nickelrich layered oxide materials usually suffer from increasingly severe capacity fading, with increasing nickel content. ${ }^{5}$ Severe electrochemical performance degradation largely originates from sharp phase transitions, causing heterogeneous lattice expansion and contraction during electrochemical cycling. ${ }^{6}$ $\mathrm{H} 2-\mathrm{H} 3$ phase transition, in a deeply delithiated state, plays a key role in determining the structural stability of a nickel-rich layered cathode, as the transformation leads to detrimentally anisotropic volume change and aggravating capacity fading.

Up to now, it remains an open question whether the degradation mechanism of Ni-rich layered cathodes is a phase transition process or a solid-solution reaction during highvoltage cycling. For example, Manthiram ${ }^{7}$ et al. reported the formation of a solid solution phase in $\mathrm{LiNi}_{0.92} \mathrm{Co}_{0.06} \mathrm{Al}_{0.02} \mathrm{O}_{2}$ electrodes during cycling, while Biasi $^{8}$ et al. demonstrated that

\footnotetext{
${ }^{a}$ School of Chemical Engineering, Sichuan University, Chengdu 610065,

P. R. China. E-mail: zhenguowu@scu.edu.cn

${ }^{b}$ Ruyuan Dongyangguang Magnetic Materials Co., Ltd, Guangdong, Ruyuan, 512000, P. R. China

${ }^{c}$ Institute for Applied Materials (IAM), Karlsruhe Institute of Technology (KIT), Hermann-von-Helmholtz-Platz 1, 76344 Eggenstein-Leopoldshafen, Germany. E-mail:weibo.hua@kit.edu

${ }^{d}$ College of Materials and Chemistry \& Chemical Engineering, Chengdu University of Technology, Chengdu, 610059, P. R. China

$\dagger$ Electronic supplementary information (ESI) available. See DOI: 10.1039/d0cc00327a
}

the $\mathrm{LiNiO}_{2}$ cathode suffers from the phase transformation from a layered hexagonal $\mathrm{H} 1$ phase to a layered $\mathrm{H} 2 / \mathrm{H} 3$ phase during the de-lithiation process. Recently, Sun's group ${ }^{9}$ reported that three phase transitions, i.e. $\mathrm{H} 1 \rightarrow \mathrm{H} 2, \mathrm{H} 2 \rightarrow \mathrm{H} 3$ and $\mathrm{H} 3 \rightarrow \mathrm{O} 1$, are detected in $\mathrm{LiNi}_{0.95} \mathrm{Co}_{0.025} \mathrm{Mn}_{0.025} \mathrm{O}_{2}$, which means that the stacking sequence of oxygen atoms changes from cubic close-packing (ABCABC) in the $\mathrm{H} 1 / \mathrm{H} 2 / \mathrm{H} 3$ phase to hexagonal close-packing $(\mathrm{ABAB})$ in the $\mathrm{O} 1$ phase when almost all Li-ions are extracted from the Li layer in the layered structure.

Here, in situ high-resolution synchrotron radiation diffraction (SRD) is used to investigate the structural evolution of a $\mathrm{LiNi}_{0.85^{-}}$ $\mathrm{Co}_{0.10} \mathrm{Mn}_{0.05} \mathrm{O}_{2}$ cathode material (N85CM). Interestingly, new phase transitions were observed, which are different from those published in the literature. ${ }^{10-13}$ Two layered phases, i.e. $\mathrm{H}_{3}$ and $\mathrm{H} 3_{b}$ phases, with the same oxygen stacking (O3 structure) but different lattice parameters are generated after charging to above $4.6 \mathrm{~V}$. To mitigate the degradation of N85CM during high-voltage cycling, small-dose of $\mathrm{Al}(2 \mathrm{~mol} \%)$ for $\mathrm{Mn}$ in $\mathrm{LiNi}_{0.85} \mathrm{Co}_{0.10} \mathrm{Mn}_{0.03} \mathrm{Al}_{0.02} \mathrm{O}_{2}$ (N85CMA) is adopted. The in situ SRD results suggest that N85CMA experienced a solid-solution reaction within a wide voltage window $\left(2.7-4.8 \mathrm{~V} v s . \mathrm{Li}^{2} \mathrm{Li}^{+}\right)$. Al-doping significantly suppresses the split hexagonal phase, proposed to mitigate the structural and functional fatigue of layered Ni-rich cathode materials during high-voltage cycling.

N85CM and N85CMA are synthesized by a sol-gel method followed by a conventional high-temperature solid-state method. ${ }^{14}$ For comparison, the traditional $\mathrm{LiCoO}_{2}$ cathode was also prepared. High-resolution synchrotron radiation diffraction (SRD) was performed (Fig. S1 in the ESI $\dagger$ ) to investigate the structures of $\mathrm{N} 85 \mathrm{CM}, \mathrm{LiCoO}_{2}$ and N85CMA. All the diffraction reflections of the three samples can be indexed to a hexagonal $\alpha-\mathrm{NaFeO}_{2}$ layered structure with a space group $R-\overline{3} m$. No obvious impurities can be observed. The electrochemical properties of the selected samples were tested at a cut-off voltage of $4.8 \mathrm{~V}$, and the initial charge-discharge curves of the electrodes are shown in Fig. 1 and Fig. S4 (ESI $\dagger$ ). Both N85CM and N85CMA electrodes are found to exhibit similar monotonous charge/discharge features. 

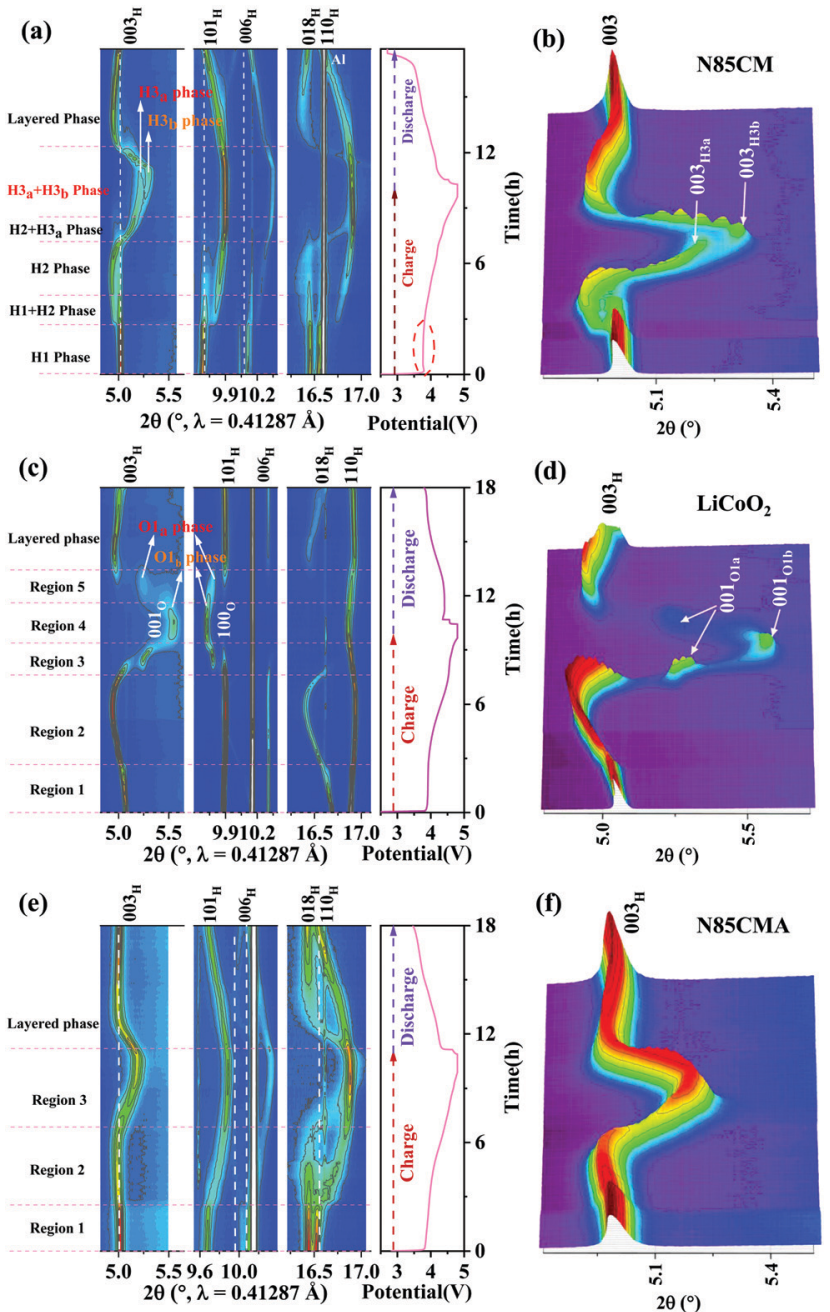

Fig. 1 In situ SRD of (a) N85CM, (c) $\mathrm{LiCoO}_{2}$ and (e) N85CMA electrodes: Contour plot of reflection evolution of 003, 101, 006, 018 and 110 during the first charging and discharging process. The original SRD patterns of 003 reflection of (b) $\mathrm{N} 85 \mathrm{CM}$, (d) $\mathrm{LiCoO}_{2}$ and (f) N85CMA during the first cycle between 2.7 and $4.8 \mathrm{~V}$. $\mathrm{H}$ and $\mathrm{O}$ indicate the hexagonal layered phase with an $\mathrm{O} 3$ structure and the trigonal layered phase with an $\mathrm{O} 1$ structure, respectively.

To illuminate the structural evolution of N85CM during high-voltage cycling, in situ high-resolution SRD was performed in the voltage range of 2.7-4.8 V. The contour plots of intensity and positions for several reflections, i.e. 003, 101, 104, 018 and 110, are exhibited in Fig. 1a. The original SRD patterns of the 003 peak during the first cycle from $2.7-4.8 \mathrm{~V}$ of $\mathrm{N} 85 \mathrm{CM}$ are exhibited in Fig. 1b, and the corresponding voltage profile of the first cycle at $2.7-4.8 \mathrm{~V}$ is illustrated in Fig. $1 \mathrm{a}$. The scattering angles of 003,104 , and 018 reflections imply the change in the average metal-metal inter-slab distance of the layered structure (i.e. $c$ lattice parameter), meanwhile 101 and 110 reflections represent the evolution of average metal-metal intra-slab distance (i.e. $a$ lattice parameter). ${ }^{15}$ Interestingly, the galvanostatic curve of N85CM exhibits a steep increase of the voltage up to $3.88 \mathrm{~V}$ in the early stage of the charge, and then the voltage decreases to yield a plateau at $3.77 \mathrm{~V}$ (marked by a red dotted cycle). Such an increase and then a decrease of the overpotential may be caused by the residual lithium on the surface of the materials, ${ }^{16}$ because there is no obvious change in the reflections on the SRD patterns (see Fig. 1a and b). Upon charging to $3.8 \mathrm{~V}$, a new set of $003,101,104$, 018, and 110 reflections occurs, indicating the appearance of a new $\mathrm{H} 2$ phase. ${ }^{17,18}$ Afterwards, the $\mathrm{H} 2$ phase remains alone until $4.3 \mathrm{~V}$ upon charging. With further de-lithiation, a group of new reflections emerges with larger deviation from previous ones, which is defined as the $\mathrm{H} 3$ a phase. Then, the $\mathrm{H} 3$ a phase grows at the expense of the $\mathrm{H} 2$ phase and the co-existence remains until 4.3-4.5 V charge. Impressively, above $4.6 \mathrm{~V}$, the characteristic reflections split again, implying a new phase with the $R \overline{3} m$ space group. The detailed SRD patterns are shown in Fig. $1 \mathrm{~b}$. The new phase with a larger deviation is identified as the $\mathrm{H} 3_{\mathrm{b}}$ phase. The coexistence of $\mathrm{H}_{3}$ and $\mathrm{H} 3_{\mathrm{b}}$ phases remains until final charging to $4.8 \mathrm{~V}$. During lithiation, the 003,104 , and 018 reflections return to lower angles. From the result above, it can be concluded that N85CM suffers from severe structural degradation during high-voltage cycling, resulting in a large hysteresis between charge and discharge processes in N85CM (e.g. the sharply decreased voltage in the beginning of the discharging process in Fig. 1a).

Each in situ SRD pattern during charging-discharging cycles has been refined by Rietveld refinement with the $R \overline{3} m$ structure model, and the corresponding lattice parameters are shown in Fig. 2a. For N85CM, at an open-circuit voltage of $3.8 \mathrm{~V}$, parameter $a$ of phase $\mathrm{H} 1$ decreases from 2.875(1) to 2.872(1) A, while parameter $c$ slightly increases from $14.203(1)$ to $14.209(1) \AA$. The contraction of parameter $a$ is supposed to be caused by the reducing radii of TM cations with higher valence states. ${ }^{19}$ The expansion of parameter $c$ is believed to be due to the increased electrostatic repulsive force between the oxygen layer and the TM layer as a result of the decreased number of Li ions in the Li layer in N85CM. ${ }^{20,21}$ From 3.8 to $4.0 \mathrm{~V}$, the lattice parameter $a$ of both $\mathrm{H} 1$ and $\mathrm{H} 2$ phases gradually decreased, the $c$ constant of the two phases increases firstly and then decreases, and the maximum values are 14.242(1) ̊ and 14.474(1) A respectively, see Fig. 2a. Compared to the $\mathrm{H} 1$ phase, a higher $c / a$ ratio indicates a better-defined crystal structure in the $\mathrm{H} 2$ phase. ${ }^{22}$ When charged to 4.3 V, parameter $a$ of the $\mathrm{H}_{3}$ a phase demonstrates a slight change and parameter $c$ rapidly decreases from $14.039(1)$ to $13.765(1) \AA$. From 4.6 to $4.8 \mathrm{~V}$, the lattice parameter $a$ of $\mathrm{H} 3_{\mathrm{a}}$ and $\mathrm{H} 3_{\mathrm{b}}$ phases remains constant within the range of experimental error, while parameter $c$ of the two layered phases separately shrinks from $13.747(1)$ to $13.728(1) \AA$ and from $13.584(1)$ to $13.526(1) \AA$. This decrease in parameter $c$ may have resulted from oxygen release and/or TM migration, leading to a reduction of the electrostatic repulsion between oxygen-oxygen layers along the $c$-axis and an expansion along the $a$ (and $b$ ) axis. The lattice parameters $(a, b$, and $c$ ) of the $\mathrm{H} 3_{\mathrm{b}}$ phase are always smaller than those of the $\mathrm{H} 3_{\mathrm{a}}$ phase, leading to a larger change in mechanical strain among various phases and thus to undesired fracture and pulverization of materials, see Fig. 3. After discharge, both parameters $a$ and $c$ do not reach the original value of the pristine material before cycling and $\Delta c$ and $\Delta a$ are 0.0609(1) A and -0.0032(1) respectively, demonstrating that the atomic structure of $\mathrm{N} 85 \mathrm{CM}$ is not totally reversible, which may result from the incomplete insertion of lithium ions into the layered structure. ${ }^{23}$ 

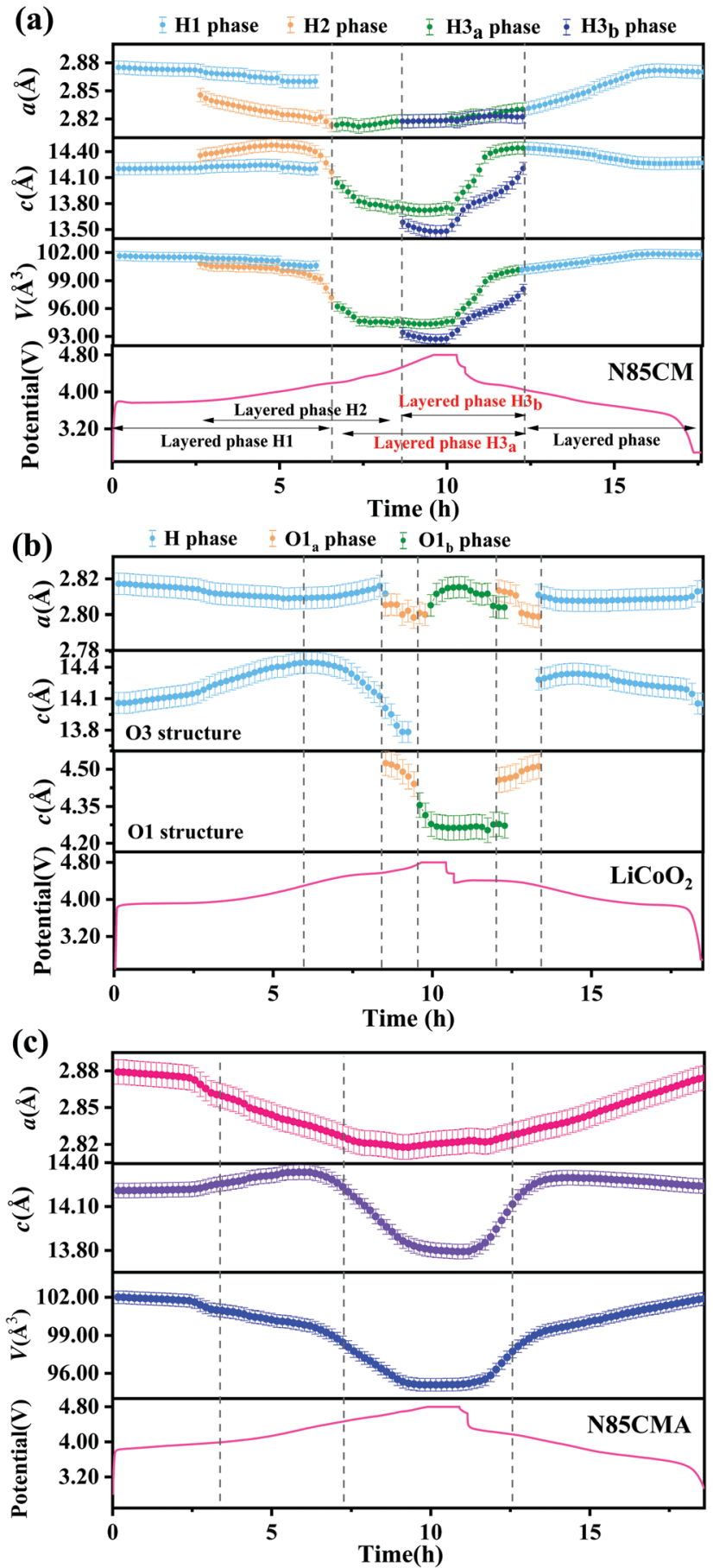

Fig. 2 Lattice parameter changes of (a) N85CM, (b) $\mathrm{LiCoO}_{2}$ and (c) N85CMA cathodes during the first charge-discharge process.

In order to clarify whether $\mathrm{O} 3$ oxygen stacking in the new layered $\mathrm{H}_{3}$ bhase is maintained, in situ SRD was performed on the traditional $\mathrm{LiCoO}_{2}$ cathode during high-voltage cycling (shown in Fig. 1(c and d)). At a highly delithiated state (4.5-4.8 V), a set of reflections such as 001 and 100 indexing to trigonal O1-type $\mathrm{Li}_{1-x} \mathrm{CoO}_{2}(x>0.8)$ is clearly probed. The observed $\mathrm{O} 1_{\mathrm{a}}$ and $\mathrm{O}_{\mathrm{b}}$ phases are in good agreement with the previous in situ XRD results of $\mathrm{LiCoO}_{2}$ reported in the literature, ${ }^{24}$ which are

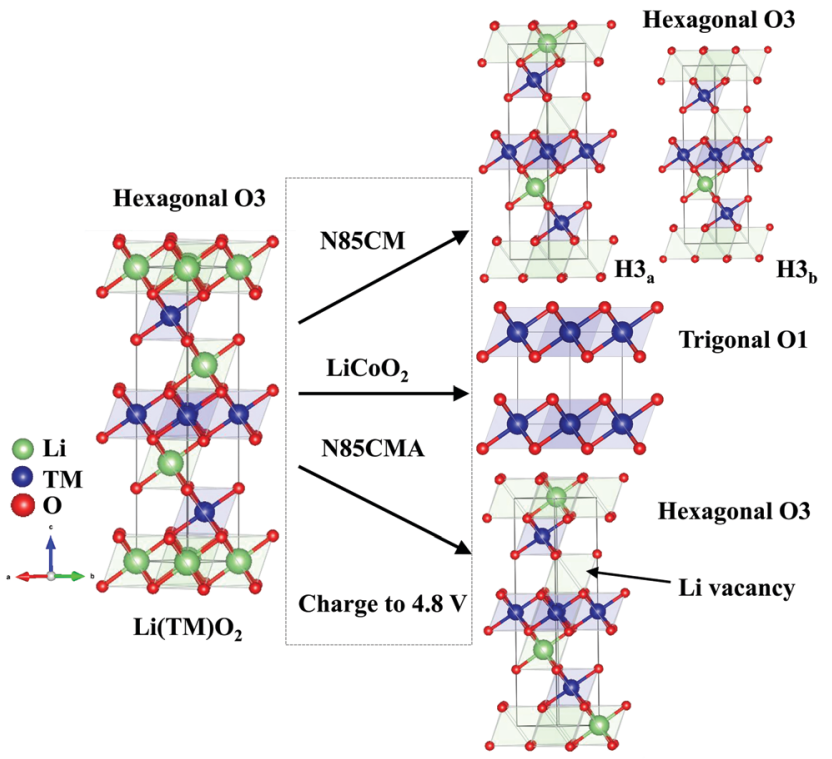

Fig. 3 Schematic diagram of phase transition during de-intercalation from O3- $\mathrm{Li}(\mathrm{TM}) \mathrm{O}_{2}$ to O3- $\mathrm{Li}_{1-x}(\mathrm{TM}) \mathrm{O}_{2}$ or $\mathrm{O} 1-\mathrm{Li}_{1-x} \mathrm{CoO}_{2}(x>0.8)$.

different with the phase transition mechanisms in N85CM. The evolution of the lattice parameters of the $\mathrm{LiCoO}_{2}$ cathode as a function of reaction time obtained from Rietveld refinement is shown in Fig. 2b, showing massive structural collapse in the $c$-axis upon high-voltage cycling.

In order to alleviate the severe structural deterioration of the N85CM material, we adopted a simple method of aluminum doping to synthesize N85CMA. Fig. 1c and Fig. S4b (ESI $\dagger$ ) show the charge-discharge curve of N85CMA during the first cycle. The voltage of N85CMA increases steeply up to about $3.68 \mathrm{~V}$ and then reaches a plateau. No pronounced increase in the voltage polarization is found in N85CMA, which matches well with those observed in the literature. ${ }^{16}$ Within a wide voltage window (2.7-4.8 $\mathrm{V}$ vs. $\mathrm{Li} / \mathrm{Li}^{+}$) and at $1.0 \mathrm{C}$ rate, $\mathrm{N} 85 \mathrm{CM}$ delivers a discharge capacity of $165.8 \mathrm{~mA} \mathrm{~h} \mathrm{~g}^{-1}$ and $57.2 \%$ capacity retention after 70 cycles, while N85CMA delivers a discharge capacity of $182.2 \mathrm{~mA} \mathrm{~h} \mathrm{~g}{ }^{-1}$ and $73.1 \%$ capacity retention after 70 cycles (Fig. S4c, ESI $\dagger$ ). After cycles, compared with N85CMA, the surface of N85CM particles suffered from severe pulverization, demonstrated in Fig. S6 (ESI $\dagger$ ). Therefore, the substitution of Al could remarkably enhance the long-term structural and chemical stability of Ni-rich layered materials, thus favoring an improved capacity of N85CMA during high-voltage cycling.

In situ high-resolution SRD measurements were performed in the voltage range of 2.7-4.8 $\mathrm{V}$ to study the structural evolution of N85CMA, as shown in Fig. 1e. The characteristic reflections 003, 101, 018 and 110 shift to lower angles firstly and then to higher angles with further de-lithiation. Besides, there is no clear reflection splitting during charging from $2.7 \mathrm{~V}$ to $4.8 \mathrm{~V}$, indicating no pronounced biphasic region. ${ }^{7}$ The continuous and steady evolution of reflections of N85CMA indicates no clear structural phase transition with cycling, suggesting that N85CMA is solid solution with the homogeneous reaction in the process of charging and discharging. ${ }^{7,25}$ Furthermore, the 
profiles of the corresponding lattice parameters of N85CMA are shown in Fig. 2c. The curves of lattice parameters $a$ and $c$ are smooth and there is no new branch of stacking axis constants. A solid solution reaction process alleviates the mechanical and structural degradation, thus resulting in a superior electrochemical performance (see Fig. S4, ESI $\dagger$ ), as also demonstrated in previous research. ${ }^{26-31}$

In summary, the structural evolution of layered Ni-rich cathode materials is investigated by high-resolution SRD. According to the results of in situ SRD, the N85CM cathode experiences complex phase transformations and a dramatically new phase transformation ( $\mathrm{H} 3_{\mathrm{a}}$ to $\mathrm{H} 3_{\mathrm{b}}$ transition). The phase transformation causes a severe change in mechanical stress among various phases, triggering off the irreversible structural degradation and functional fatigue. It is found that the substitution of Al could effectively suppress the $\mathrm{H} 2-\mathrm{H} 3$ phase transformation, beneficial to the improvement of the electrochemical performance of Ni-rich cathode materials. With this new exploration and effective modification, more nickel rich cathode materials with excellent structural stability and electrochemical performance could be expected in the near future.

This work was supported by the National Natural Science Foundation of China (No. 21805198, 21878195, 21805018), the Youth Foundation of Sichuan University (No. 2017SCU04a08), the National Key Research and Development of China (grant no. 2016YFD0200404), and the Research Foundation for the Postdoctoral Program of Sichuan University (No. 2017SCU12018 and 2018SCU12045). W. H. received financial support from the Helmholtz - OCPC Postdoc-Program. Parts of this research were carried out at ALBA Light Source using MSPD beamline. We thank Dr Zhuo Zheng for help with data analysis. This work contributes to the research performed at CELEST (Center for Electrochemical Energy Storage Ulm-Karlsruhe).

\section{Conflicts of interest}

There are no conflicts to declare.

\section{Notes and references}

1 W. Hua, S. Wang, M. Knapp, S. J. Leake, A. Senyshyn, M. Yavuz, J. R. Binder, C. P. Grey, H. Ehrenberg, S. Indris and B. Schwarz, Nat. Commun., 2019, 24, 1-11.

2 J. Y. Liao and A. Manthiram, J. Power Sources, 2015, 282, 429-436.

3 W. Dong, X. X. Zeng, X. D. Zhang, J. Y. Li, J. L. Shi, Y. Xiao, Y. Shi, R. Wen, Y. X. Yin, T. S. Wang, C. R. Wang and Y. G. Guo, ACS Appl. Mater. Interfaces, 2018, 10, 18005-18011.

4 Y. Xiao, P. F. Wang, Y. X. Yin, Y. F. Zhu, Y. B. Niu, X. D. Zhang, J. Zhang, X. Yu, X. D. Guo, B. H. Zhong and Y. G. Guo, Adv. Mater., 2018, 30, 1803765.
5 X. Q. Yang, X. Sun and J. McBreen, Electrochem. Commun., 1999, 1, 227-232.

6 U. H. Kim, H. H. Ryu, J. H. Kim, R. Mucke, P. Kaghazchi and Y. K. Sun, Adv. Energy Mater., 2019, 9, 18.

7 J. Y. Li, W. D. Li, S. Y. Wang, K. Jarvis, J. H. Yang and A. Manthiram, Chem. Mater., 2018, 30, 3101-3109.

8 L. D. Biasi, A. Schiele, R.-A. Maria, G. Garcia, T. Brezesinski, P. Hartmann and J. Janek, ChemSusChem, 2019, 12, 2240.

9 U.-H. Kim, H.-H. Ryu, J.-H. Kim, R. Mücke, P. Kaghazchi, C. S. Yoon and Y.-K. Sun, Adv. Energy Mater., 2019, 1803902.

10 T. Chen, X. Li, H. Wang, X. X. Yan, L. Wang, B. W. Deng, W. J. Ge and M. Z. Qu, J. Power Sources, 2018, 374, 1-11.

11 T. Li, X. H. Li, Z. X. Wang and H. J. Guo, J. Power Sources, 2017, 342, 495-503.

12 A. Manthiram, J. C. Knight, S. T. Myung, S. M. Oh and Y. K. Sun, Adv. Energy Mater., 2016, 6, 1.

13 W. Liu, P. Oh, X. Liu, M. J. Lee, W. Cho, S. J. Chae, Y. Kim and J. Cho, Angew. Chem., Int. Ed., 2015, 54, 4440-4457.

14 S. W. Lee, H. Kim, M. S. Kim, H. C. Youn, K. Kang, B. W. Cho, K. C. Roh and K. B. Kim, J. Power Sources, 2016, 315, 261-268.

15 W. Hua, B. Schwarz, M. Knapp, A. Senyshyn, A. Missiul, X. K. Mu, S. N. Wang, C. Kubel, J. R. Binder, S. Indris and H. Ehrenberg, J. Electrochem. Soc., 2019, 166, A5025-A5032.

16 Y. You, H. Celio, J. Li, A. Dolocan and A. Manthiram, Angew. Chem., Int. Ed., 2018, 57, 6480.

17 Y. Xia, J. M. Zheng, C. Wang and M. M. Gu, Nano Energy, 2018, 49, $434-452$.

18 J. L. Shi, D. D. Xiao, M. Ge, X. Yu, Y. Chu, X. Huang, X. D. Zhang, Y. X. Yin, X. Q. Yang, Y. G. Guo, L. Gu and L. J. Wan, Adv. Mater., 2018, 30, 1705575.

19 Y. W. Tsai, B. J. Hwang, G. Ceder, H. S. Shen, D. G. Liu and J. F. Lee, Chem. Mater., 2005, 17, 3191-3199.

20 J. Li, E. L. Downie, L. Ma, W. D. Qiu and J. R. Dahn, J. Electrochem. Soc., 2015, 162, A1401-A1408.

21 Y. Xiao, Y. F. Zhu, H. R. Yao, P. F. Wang, X. D. Zhang, H. L. Li, X. N. Yang, L. Gu, Y. C. Li, T. Wang, Y. X. Yin, X. D. Guo, B. H. Zhong and Y. G. Guo, Adv. Energy Mater., 2019, 9, 1803978.

22 J. Li, N. Zhang, H. Y. Li, A. Liu, Y. Q. Wang, S. Yin, H. H. Wu and J. R. Dahn, J. Electrochem. Soc., 2018, 165, A3544-A3557.

23 A. O. Kondrakov, A. Schimidt, J. Xu, H. Geßwein, R. Mönig, P. Hartmann, H. Sommer, T. Brezesinski and J. Janek, J. Phys. Chem. C, 2017, 121, 3286-3294.

24 K. Y. Chung, W. S. Yoon, H. S. Lee, J. McBreen, X. Q. Yang, S. H. Oh, W. H. Ryu, J. L. Lee, W. I. Cho and B. W. Cho, J. Power Sources, 2006, 163, 185-190.

25 Y. Xiao, P. F. Wang, Y. X. Yin, Y. F. Zhu, X. N. Yang, X. D. Zhang, Y. S. Wang, X. D. Guo, B. H. Zhong and Y. G. Guo, Adv. Energy Mater., 2018, 8, 1800492.

26 W. B. Hua, X. D. Guo, Z. Zheng, Y. J. Wang, B. H. Zhong, B. Z. Fang, J. Z. Wang, S. L. Chou and H. Liu, J. Power Sources, 2015, 275, 200-206.

27 J. L. Shi, D. D. Xiao, X. D. Zhang, Y. X. Yin, Y. G. Guo, L. Gu and L. J. Wan, Nano Res., 2017, 10, 4201-4209.

28 J. L. Shi, J. N. Zhang, M. He, X. D. Zhang, Y. X. Yin, H. Li, Y. G. Guo, L. Gu and L. J. Wan, ACS Appl. Mater. Interfaces, 2016, 8, 20138-20146.

29 Y. Xiao, X. D. Zhang, Y. F. Zhu, P. F. Wang, Y. X. Yin, X. N. Yang, J. L. Shi, J. Liu, H. L. Li, X. D. Guo, B. H. Zhong and Y. G. Guo, Adv. Sci., 2019, 6, 1801908.

30 J. L. Shi, R. Qi, X. D. Zhang, P. F. Wang, W. G. Fu, Y. X. Xu, J. Xu, L. J. Wan and Y. G. Guo, ACS Appl. Mater. Interfaces, 2017, 9, 42829-42835.

31 W. Hua, M. Chen, B. Schwarz, M. Knapp, M. Bruns, J. Barthel, X. Yang, F. Sigel, R. Azmi, A. Senyshyn, A. Missiul, L. Simonelli, M. Etter, S. Wang, X. Mu, A. Fiedler, J. R. Binder, X. Guo, S. Chou, B. Zhong, S. Indris and H. Ehrenberg, Adv. Energy Mater., 2019, 9, 1803094. 